\title{
Conformation-dependent GAD65 autoantibodies in diabetes
}

\author{
D. Luo ${ }^{1}$ L. K. Gilliam ${ }^{1}$ C. Greenbaum ${ }^{2}$ L. Bekris ${ }^{1}$ C. S. Hampe ${ }^{1}$ T. Daniels ${ }^{1}$ W. Richter ${ }^{3}$ \\ S. M. Marcovina ${ }^{1}$ - O. Rolandsson ${ }^{4} \cdot$ M. Landin-Olsson ${ }^{5} \cdot$ I. Kockum$^{6} \cdot$ A. Lernmark $^{1}$ \\ ${ }^{1}$ Department of Medicine, University of Washington, R. H. Williams Laboratory, Seattle, USA \\ ${ }^{2}$ Benaroya Research Institute, Seattle, Washington, USA \\ ${ }^{3}$ Department of Orthopaedic Surgery, University of Heidelberg, Heidelberg, Germany \\ ${ }^{4}$ Department of Public Health and Clinical Medicine, University of Umeå, Sweden \\ ${ }^{5}$ Department of Medicine, Lund University Hospital, Lund, Sweden \\ ${ }^{6}$ Department of Molecular Medicine, Karolinska Institute, Stockholm, Sweden
}

\section{Abstract}

Aims/hypothesis. Conformation-dependent autoantibodies directed against GAD65 are markers of Type 1 diabetes. In this study we aimed to determine whether the substitution of GAD65 with GAD67 amino acids would affect the binding of conformation-dependent GAD65 autoantibodies.

Methods. We used PCR-based site-directed mutagenesis to generate a series of mutated GAD65 cDNA constructs in which specific GAD65 coding sequences for regions of the protein critical for autoantibody binding were replaced with GAD67 coding sequences.

Results. The introduction of a point mutation at position 517, substituting glutamic acid with proline, markedly reduced the binding of disease-associated GAD65 antibodies. The binding of GAD65 antibodies to the E517P mutant was reduced in the sera of all newly diagnosed Type 1 diabetes patients $(n=85)$ by a mean of $72 \%(p<0.0001)$ compared with binding to wild-type GAD65. Patients with latent autoimmune diabetes in adults $(n=24)$ showed a similar reduction in binding (79\% reduction, $p<0.0001)$. First-degree relatives who subsequently progressed to Type 1 diabetes $(n=12)$ showed a reduction in binding of $80 \%$ compared with a reduction of only $65 \%$ among relatives who had not progressed to disease $(n=38$; $p=0.025)$. In healthy GAD65Ab-positive individuals who did not progress to diabetes during a 9-year follow-up period $(n=51)$, binding to GAD65-E517P was reduced by only $28 \%$ compared with binding to wildtype GAD65.

Conclusions/interpretation. Differences in autoantibody binding to wild-type GAD65 versus GAD65E517P may provide predictive information about Type 1 diabetes risk beyond that provided by the presence or absence of GAD65 autoantibodies. Lack of binding to mutant GAD65-E517P defines GAD65positive individuals who are at higher risk of developing diabetes.

Keywords Autoantibody - Autoimmunity - Epitope · GAD65 - Latent autoimmune diabetes in adults .

Type 1 diabetes . Type 2 diabetes
Received: 27 February 2004 / Accepted: 30 June 2004

Published online: 8 September 2004

(C) Springer-Verlag 2004

Å. Lernmark (凶)

Department of Medicine, University of Washington,

R. H. Williams Laboratory, 1959 N. E. Pacific Street,

Box 357710, Seattle, WA 98195-7710, USA

E-mail: ake@u.washington.edu

Tel.: +1-206-5435316, Fax: +1-206-5433169

Abbreviations: GAD65Ab, GAD65 antibody · IAA, insulin autoantibody $\cdot$ IA-2, islet antigen- $2 \cdot$ IA-2Ab, islet antigen-2 antibody - ICA, islet cell antibody $\cdot \mathrm{LADA}$, latent autoimmune diabetes in adults - mAb144, mouse monoclonal antibody 144 . MICA, monoclonal islet cell antibody $\cdot$ SMS, stiff-man syndrome

\section{Introduction}

Type 1 diabetes develops following the selective immunological destruction of pancreatic islet beta cells, leading to insulin deficiency. Several targets of the autoimmune process have been identified, including GAD65. Unlike GAD67, GAD65 is a major self-antigen in Type 1 diabetes, and GAD65 autoantibodies represent early markers of beta cell autoimmunity (reviewed in [1]). The two GAD isoforms are highly homologous and differ mostly in the amino-terminal third of the protein [2], a feature useful for identifying disease-associated epitopes [3, 4]. Positivity for the GAD65 antibody (GAD65Ab) confers high diagnostic 
sensitivity for Type 1 diabetes (80\%) [5]. For this reason, GAD65Ab are currently one of the markers used to identify non-diabetic subjects at risk of developing Type 1 diabetes. However, the diagnostic specificity of GAD65Ab is limited by the presence of these antibodies in about $1 \%$ of healthy individuals $[6,7]$ and in patients with other autoimmune disorders, including stiff-man syndrome (SMS) [8]. Even in first-degree relatives, GAD65Ab positivity alone is not a perfect predictor of progression to disease. In the Seattle Family Study, GAD65Ab positivity in relatives who also tested positive for islet cell antibody (ICA) or insulin autoantibody (IAA) conferred a moderate (50\%) 5-year risk of developing Type 1 diabetes [9], compared with a much higher (nearly 100\%) 5-year risk conferred by the presence of all three autoantibodies [10].

Type 1 diabetes sera primarily recognise conformational GAD65 epitopes, and GAD65Ab binding is easily lost when the target antigen is denatured, such as in ELISA and western blots or following mutagenesis $[11,12,13]$. The ability to identify diabetes-specif- ic GAD65Ab that recognise conformation-dependent epitopes would enhance the value of GAD65Ab as a predictive marker. Several approaches have been used to identify GAD65Ab disease-specific epitopes, including deletion constructs [14], chimeric GAD65/67 molecules $[15,16]$ and naturally occurring GAD65 isoforms [17]. The majority of data suggest that conformation-dependent epitopes are found at the middle and at the C-terminal end of GAD65 [12, 15, 16, 18]. We previously demonstrated that GAD65Ab that bind to the C-terminal end of GAD65 distinguished children with Type 1 diabetes from healthy children [18]. Similarly, the presence of GAD65Ab that bind to C-terminal epitopes in patients with latent autoimmune diabetes in adults (LADA) identified a subgroup of individuals with low BMI, low basal C-peptide values and an earlier requirement for insulin therapy [16]. The aim of the present study was to determine whether the alteration of a C-terminal GAD65Ab epitope, by substituting GAD67-derived amino acids, defines GAD65Ab with increased diagnostic specificity for Type 1 diabetes.

Table 1. The GAD65-specific antibody reagents used to determine the effects of GAD65 mutations on antibody binding

\begin{tabular}{|c|c|c|c|}
\hline Group & Antibody & Antigen & Reference \\
\hline \multirow[t]{5}{*}{ A. Monoclonal GAD65 antibodies } & mAb144 & GAD65 4-22 peptide (mouse) & [19] \\
\hline & GAD6 & Purified GAD65 (mouse) & {$[20]$} \\
\hline & MICA2 & T1DM pt, M, 32 years & {$[11,21]$} \\
\hline & MICA3 & T1DM pt, M, 32 years & {$[11,21]$} \\
\hline & MICA4 & T1DM pt, M, 32 years & {$[11,21]$} \\
\hline \multirow[t]{6}{*}{ B. Human reference sera } & 591 & T1DM pt, F, 13 years, DR3/4 & {$[41]$} \\
\hline & 613 & T1DM pt, M, 12 years, DR3/4 & [41] \\
\hline & 622 & T1DM pt, M, 15 years, DR3/4 & [41] \\
\hline & 652 & T1DM pt, M, 14 years, DR3/4 & [41] \\
\hline & $673 *$ & T1DM pt, F, 11 years, DR3/4 & {$[41]$} \\
\hline & 686 & T1DM pt, F, 10 years, DR3/4 & {$[41]$} \\
\hline \multirow[t]{12}{*}{ C. GAD65 peptide rabbit antisera } & \#7222 & GAD65 amino acids 73-91 & {$[23]$} \\
\hline & \#7641 & GAD65 amino acids 250-269 & [23] \\
\hline & \#8090 & GAD65 amino acids 250-269 & [23] \\
\hline & \#8100 & GAD65 amino acids 250-269 & {$[23]$} \\
\hline & \#5545 & GAD65 amino acids 390-404 & [23] \\
\hline & \#5052 & GAD65 amino acids 390-403 & {$[23]$} \\
\hline & \#5551 & GAD65 amino acids 390-404 & [23] \\
\hline & \#5576 & GAD65 amino acids 390-403 & [23] \\
\hline & \#5581 & GAD65 amino acids 390-403 & [23] \\
\hline & \#5482 & GAD65 amino acids $405-418$ & [23] \\
\hline & \#5547 & GAD65 amino acids $405-418$ & {$[23]$} \\
\hline & \#5571 & GAD65 amino acids $405-418$ & [23] \\
\hline
\end{tabular}

* Serum 673 is the World Health Organization standard for ICA and GAD65Ab [42]. F, female; M, male; T1DM pt, Type 1 diabetes mellitus patient 


\section{Subjects and methods}

GAD65 antibodies. Three types of GAD65-specific antibody reagents were used (Table 1). Group A included: (i) mouse monoclonal antibody 144 (mAb144), specific for the $\mathrm{N}$ terminus of GAD65 [19]; (ii) mouse monoclonal antibody GAD6, which recognises the $\mathrm{C}$ terminus of GAD65 [20]; and (iii) human monoclonal islet cell antibodies (MICA: MICA1, MICA2, MICA3, MICA4 and MICA6) [11, 17, 21]. Group B included standard sera from ten children (aged 11-16 years) newly diagnosed with Type 1 diabetes, which were obtained at the clinical onset of disease [22]. Group C included 14 rabbit antisera previously generated by immunisation with synthetic peptides specific for human GAD65 [23]. Antibody specificities are shown in Table 1.

Patients and control subjects. We studied sera from six groups of GAD65Ab-positive individuals. Sera that reacted with both GAD65 and GAD67 were not included, since they represent less than 15 to $20 \%$ of sera from newly diagnosed Type 1 diabetes patients [18]. The groups included newly diagnosed Type 1 diabetes patients $(n=85)$ and patients with LADA $(n=24)$ who were registered in the Diabetes Incidence Study in Sweden [24, 25]. We also studied GAD65Ab-positive firstdegree relatives identified or followed through the Diabetes Prevention Trial-NW Clinical Center, the Juvenile Diabetes Research Foundation Translational Research Study, and the Seattle Family Study, who had either progressed $(n=12)$ or not progressed $(n=38)$ to disease onset $[4,9]$. Among the group of "progressors", the mean follow-up time before diabetes onset was $2.5 \pm 1.3$ years. The "non-progressors" were followed-up for a period of between 1 and 10 years. We also analysed GAD65Ab-positive healthy subjects $(n=51)$ who had remained non-diabetic during 9 years of follow-up [6] from a study that screened a population-based cohort of 2157 healthy adults [7]. Finally, we analysed sera from SMS patients $(n=4)$ [8]. Demo- graphic data for all study subjects are shown in Table 2. Informed consent was obtained from all subjects and the investigations were carried out in accordance with the principles of the Declaration of Helsinki, as revised in 2000.

PCR-mediated mutagenesis. Comparison of the amino acid sequences of GAD65 and GAD67 at the C-terminal region indicated a marked sequence dissimilarity from amino acid residues 515 to 525 (Table 3 ). The remainder of the $\mathrm{C}$-terminal region shared approximately $82 \%$ sequence identity. A panel of GAD65/67 chimeras was generated to cover this small region of 11 amino acid residues (Table 3 ). The mutagenic PCR primers used to generate a point mutation substituting glutamic acid with proline at position 517 to produce the construct GAD65-E517P are shown below (mutations underlined):

\section{DL-1: $\quad$ 5'-GTATAAGATCTGGATGCATG-3' (contains a $B g l \mathrm{II}$ restriction site) \\ DL-2: 5'-GCCCTCTAGAGAAGTGGAACAG-3'

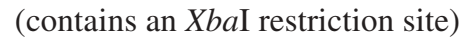 \\ DL-3: 5'-GCGTACTCTGCCAGACAATGAAG-3'

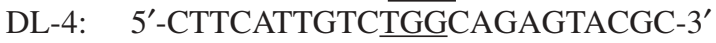

Plasmid pEx9-GAD65 cDNA (1 pg) was used for PCR amplification by a standard procedure, according to the manufacturer's instructions (Stratagene, La Jolla, Calif., USA) [3]. The PCR products were then analysed on agarose gels. Plasmid pEx9-GAD65-E517P was generated by replacing $B g l \mathrm{II} / \mathrm{XbaI}$ fragments of pEx9 with PCR-amplified DNA products containing base substitutions generated by the PCR-mediated mutagenesis. All constructs (Table 3) were sequenced for verification of the point mutations.

Coupled in vitro transcription/translation and radioimmunoassay. ${ }^{35}$ S-labelled GAD65, its variant molecules, and islet antigen-2 (IA-2) were prepared by in vitro transcription/translation

Table 2. Demographics of the study populations

\begin{tabular}{lccc}
\hline Study population & $N$ & Age $^{\mathrm{a}}$ & Sex (men/women) \\
\hline Type 1 diabetes patients & 85 & $9.5(1-16)$ & $47 / 53$ \\
LADA patients & 24 & $44.6(28-62)$ & $56 / 44$ \\
First-degree relatives & 50 & & $12.3(6-17)$ \\
$\quad$ Progressor $(n=12)$ & & $39.4(8-74)$ & $57 / 43$ \\
$\quad$ Non-progressor $(n=38)$ & 51 & $50(30-60)$ & $62 / 38$ \\
Healthy control subjects & 4 & $49.3(30-65)$ & $42 / 58$ \\
SMS patients & & $25 / 75$ \\
\hline
\end{tabular}

a Values shown are means (ranges); ${ }^{b}$ values shown are $\%$

Table 3. GAD constructs and GAD antibody reactivity

\begin{tabular}{|c|c|c|c|}
\hline \multirow[t]{2}{*}{ Name of construct } & \multirow[t]{2}{*}{ GAD65 515-525 sequence } & \multicolumn{2}{|c|}{ Binding with antibodies } \\
\hline & & Group $\mathrm{A}^{\mathrm{a}}$ & Group B \\
\hline GAD67 & GVP-SPQ-REK & - & - \\
\hline GAD65-REK & -...-REK & + & + \\
\hline GAD65-GVP & GVP-_-... & - & - \\
\hline
\end{tabular}

Group A and B antibodies are summarised in Table 1. a Excluding the mAb144 antibody. +, binding; -, no binding 


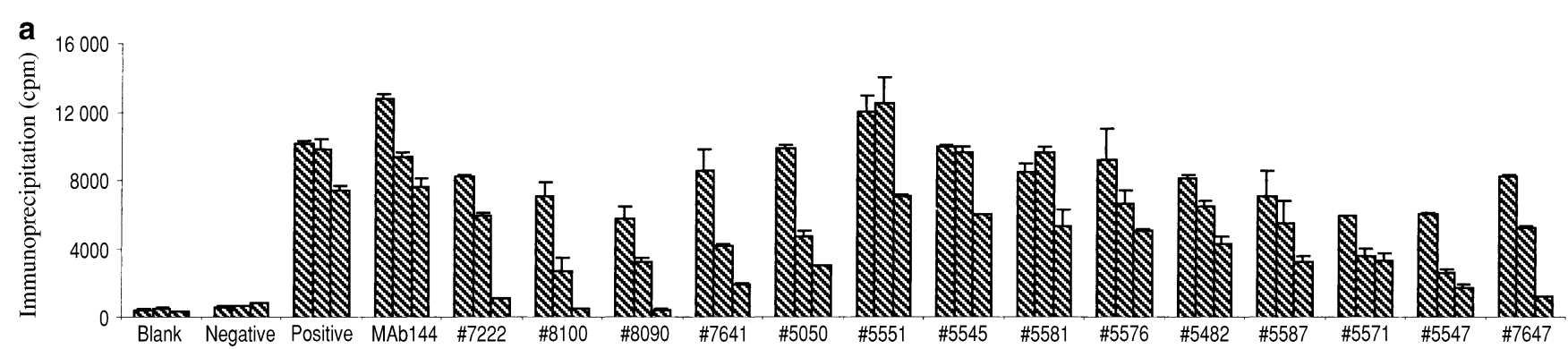

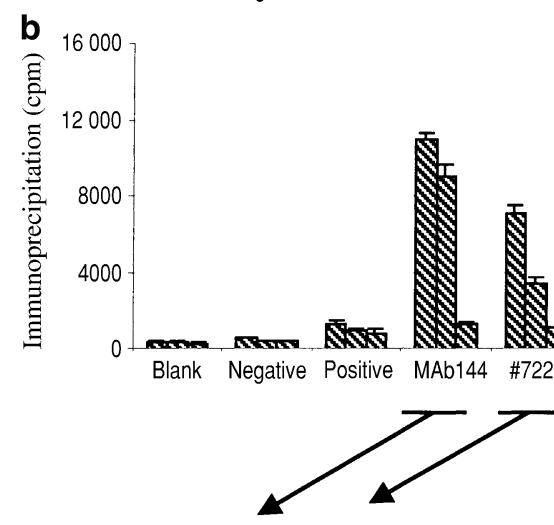

Position: 4-22 73-91
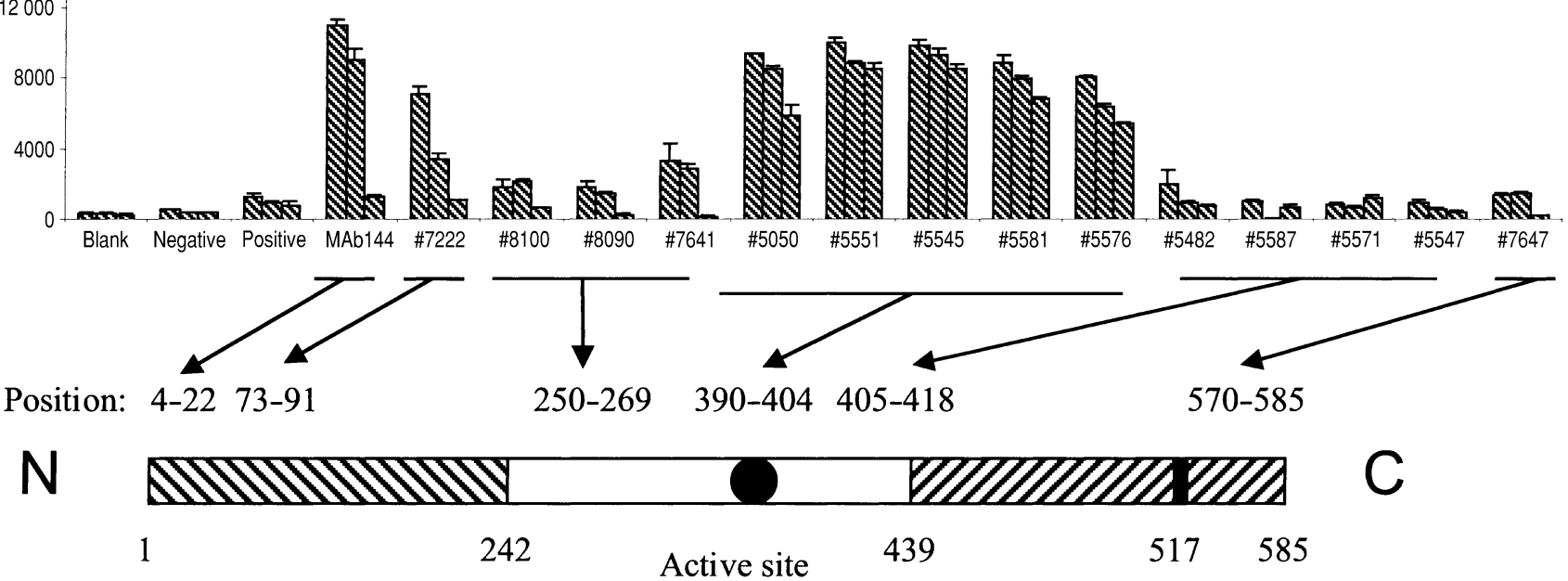

Fig. 1. GAD65Ab binding to wild-type GAD65 (a) and E517P-GAD65 (b). The GAD65Ab radioligand binding assay was used to analyse the reactivity of polyclonal rabbit antisera directed against different peptides of the GAD65 molecule (as indicated in the diagram at the bottom). The results were compared with those for mAb144 directed against the N-terminal end (residues 4-22) of GAD65, a GAD65Ab-positive serum from a Type 1 diabetes patient (positive), assay buffer alone (blank) and a GAD65Ab-negative human serum (negative). Values are shown as means \pm SEM for three independent analyses carried out at three different dilutions (1:100, 1:200 and 1:400). Some error bars are too small to show

reactions with SP6 RNA polymerase and rabbit reticulocyte lysate (Promega, Madison, Wis., USA), according to the manufacturer's instructions [3]. The binding activity of GAD65 and IA-2 was determined by Protein A-Sepharose-mediated RIA as previously described $[3,26]$. GAD65Ab and IA-2 antibody (IA-2Ab) indices were calculated as previously described [3], using the World Health Organization (WHO)/Juvenile Diabetes Foundation International (JDfI) standard as a positive control [27].

Statistical analysis. Values are shown as means \pm SD or means \pm SEM as indicated. As the antibody binding levels passed the normality test, we used the Student's $t$ test to analyse differences between groups of GAD65Ab-positive subjects. The chi square test with Yate's correction was used for differences in frequencies. A $p$ value of less than 0.05 was considered statistically significant.

\section{Results}

Sequences important for GAD65 antibody binding to GAD65 molecules. We observed no binding of mouse or human GAD65 monoclonal antibodies (Group A, Table 1) or reference sera from Type 1 diabetes patients (Group B, Table 1) to GAD67, GAD65-GVP (data not shown) or GAD65-E517P. In contrast, binding of these antibodies to GAD65-REK and GAD65-E517A was intact, similar to the binding to wild-type GAD65 (Table 3). This suggests that the specific sequences required for conformation-dependent GAD65Ab binding were abrogated by substituting glutamic acid with proline at residue 517. The absence of an effect by substituting glutamic acid with alanine suggests that the proline residue is key to the disruption of the conformational epitope recognised by GAD65Ab-positive Type 1 diabetes sera.

Analysis of GAD65-reactive rabbit polyclonal antisera, monoclonal antibodies, and human polyclonal sera. To analyse GAD65Ab epitopes sensitive to the GAD65-E517P mutation and to define possible interactive sequences in other GAD65 regions, we analysed the binding of rabbit polyclonal antisera (Group C, Table 1) directed against GAD65-specific peptides [23]. As shown in Figure 1, the E517P point mutation reduced GAD65 Ab binding to the C-terminal and middle regions, but not to the $\mathrm{N}$ terminus (amino acid residues 4-91) or to the GAD65 enzymat- 


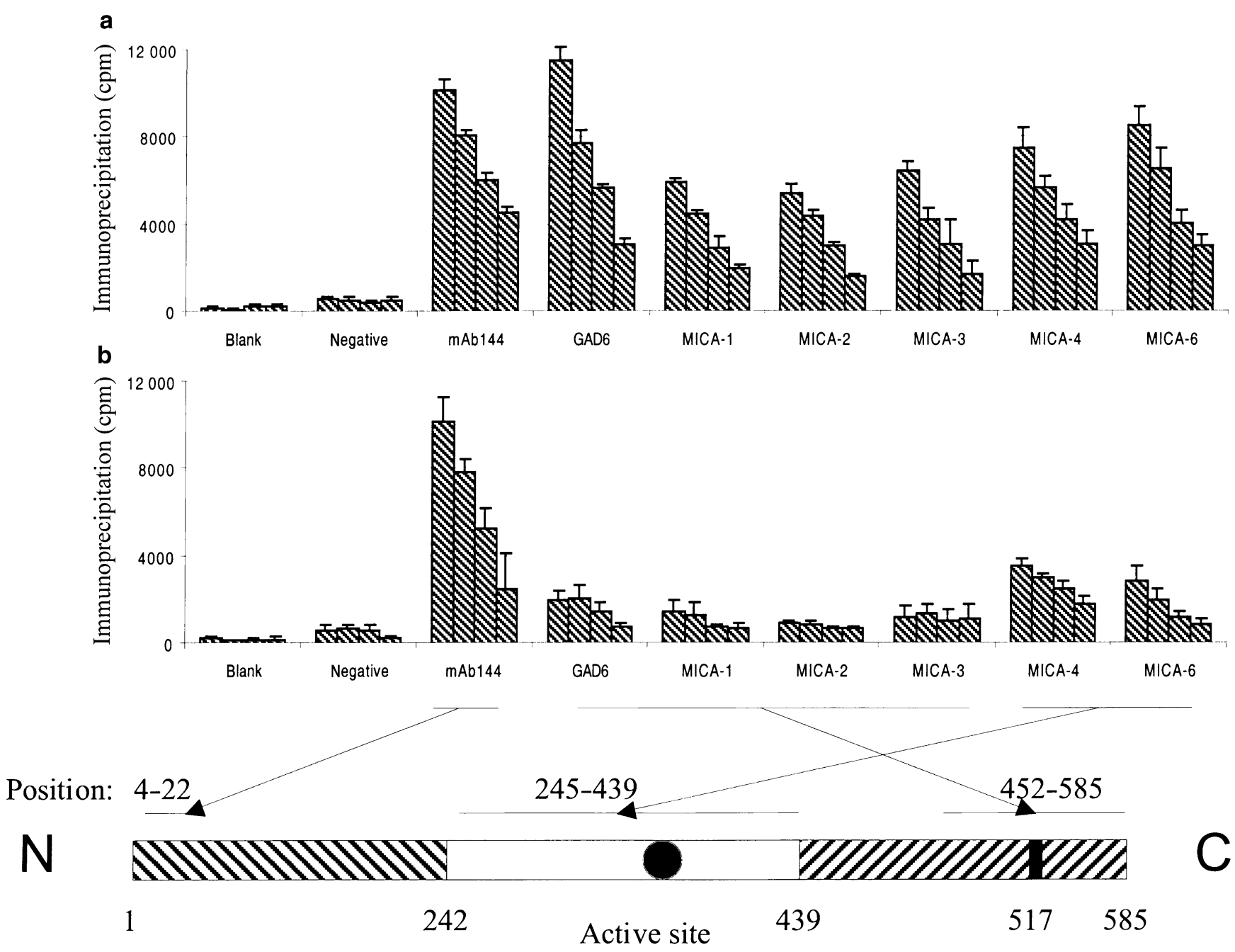

Fig. 2. GAD65Ab binding to wild-type GAD65 (a) and E517P-GAD65 (b). The GAD65Ab radioligand binding assay was used to analyse the reactivity of MICA1, MICA2, MICA3, MICA4 and MICA6, which were derived from a patient with Type 1 diabetes (directed against different regions of the GAD65 molecule as indicated in the diagram at the bottom). The results were compared with those for mAb144, mouse monoclonal antibody GAD6, assay buffer alone (blank) and a GAD65Ab-negative human serum (negative). Values are means \pm SEM for three independent analyses carried out at four different dilutions (1:50,1:100, 1:200 and 1:400). Some error bars are too small to show

ic active site (390-404). Within the middle region of the protein (250-269) and downstream of the active site (405-418), the GAD65-E517P mutation induced a marked reduction in antibody binding (Fig. 1). This suggests that amino acid residues in the middle and $\mathrm{C}$-terminal regions of the protein interact to form a conformation-dependent GAD65Ab epitope that is easily destroyed by substituting glutamic acid with proline at position 517 .

We compared the binding of wild-type GAD65 and GAD65-E517P by GAD65 monoclonal antibodies recognising different regions of the GAD65 molecule
(Group A, Table 1), including mAb144 [19], GAD6 [20] and MICA [11, 21] (Fig. 2). The binding of $\mathrm{mAb} 144$, which is specific to the $\mathrm{N}$ terminal, was not affected by the GAD65-E517P mutation. In contrast, the E517P mutation reduced the binding of GAD6, MICA1-4 and MICA 6 by between 52 and $83 \%$. Compared with the reductions observed for the other MICAs, the reductions in the binding to GAD65E517P by MICA4 and MICA6 were more moderate, consistent with previous findings that these monoclonal antibodies recognise sequences upstream of the enzymatic active site [12, 21].

We next analysed 214 GAD65Ab-positive human sera from the six groups of subjects described in the methods section. Autoantibody binding to GAD65E517P was compared with binding to wild-type GAD65 (100\% binding) under identical assay conditions. The GAD65Ab binding patterns for the six groups were very different (Fig. 3a). Binding to GAD65-E517P was markedly reduced in the Type 1 diabetes sera: the mean binding was $28 \pm 11 \%$ of the binding to wild-type GAD65 $(p<0.0001)$, equivalent to a $72 \%$ average reduction in binding. The reduction in binding ranged from $47 \%$ to $90 \%$ and was independent of the GAD65Ab titre (data not shown). A cut- 

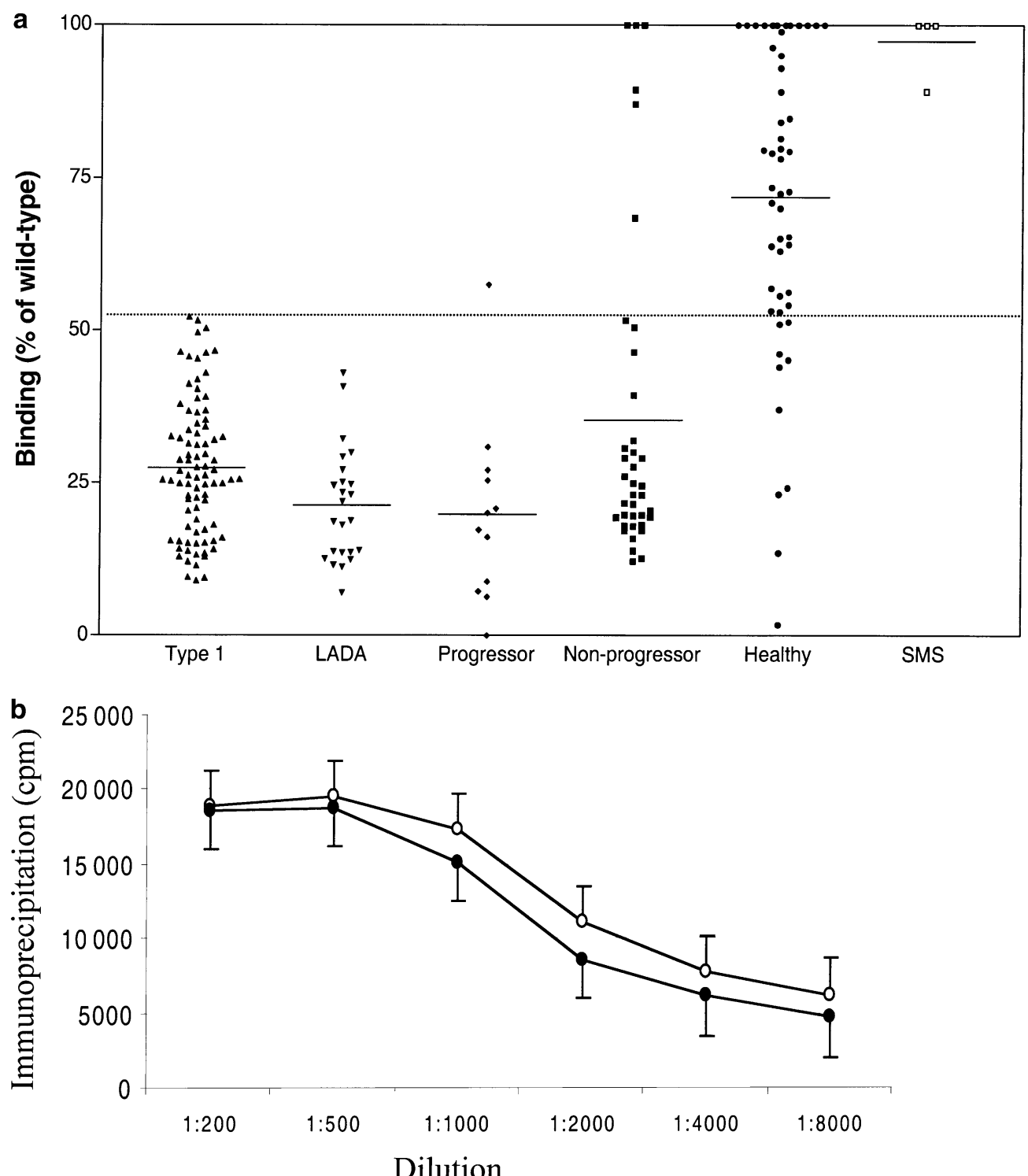

Fig. 3. GAD65Ab binding to GAD65-E517P compared with wild-type GAD65 (100\%) in six groups of serum samples. a. Individual binding levels are shown for GAD65Ab-positive Type 1 diabetes patients $(n=85)$, patients with LADA $(n=24)$, first-degree relatives who subsequently progressed to Type 1 diabetes $(n=12)$, first-degree relatives who did not progress to Type 1 diabetes $(n=38)$, healthy control subjects $(n=51)$ and patients with SMS $(n=4)$. Mean values are indicated with horizontal lines. The dotted line indicates the "positivity" cut-off value, defined as the mean plus two standard deviations of the reduction in binding to GAD65-E517P among the Type 1 diabetes patients. b. Comparison of the binding of GAD65Ab autoantibodies to the different GAD65 molecules in patients with SMS $(n=4)$. All SMS sera were tested at a range of dilutions between 1:250 and 1:8000 with both wild-type GAD65 (O) and GAD65-E517P (O). Mean values \pm SEM for four independent analyses are shown

off value of $53 \%$, defined as the mean percentage binding plus two standard deviations (indicated by a dotted horizontal line in Fig. 3a), delineated the upper limit of the Type 1 diabetes group in terms of percentage binding to GAD65-E517P compared with binding to wild-type GAD65. These data demonstrate that all Type 1 diabetes sera tested were sensitive to the E517P mutation.

This finding was further supported by the results for the LADA patients, whose sera showed a significant reduction in GAD65Ab binding to GAD65E517P (mean binding 21 $\pm 9 \%, 79 \%$ reduction) similar to that seen in Type 1 diabetes patients (Fig. 3a). The sera of all 24 LADA patients showed a reduction in binding to GAD65-E517P that was less than 53\% of the binding to wild-type GAD65. Among the 38 GAD65Ab-positive first-degree relatives who had not progressed to disease ("non-progressors"), GAD65E517P reduced GAD65Ab binding by an average of $65 \%$ (mean binding $35 \pm 26 \%$ ). The results of GAD65E517P binding by this group were subject to greater variation than the results of the other groups. While GAD65-E517P reduced GAD65Ab binding in 32 of the 38 sera to levels below the cut-off value of $53 \%$, the results of six sera were above the cut-off value, and three sera showed no reduction in GAD65Ab 
binding to the mutant GAD65 molecule. In comparison, the 12 relatives who later progressed to Type 1 diabetes ("progressors") showed a significant reduction in GAD65Ab binding to GAD65-E517P (mean binding $20 \pm 15 \%, 80 \%$ reduction). The reduction in binding was significantly different between the progressors and the non-progressors $(p=0.025)$. A much smaller reduction in binding to GAD65-E517P was observed among the 51 GAD65Ab-positive healthy control subjects (mean binding $72 \pm 25 \%, 28 \%$ reduction). Among this group, 41 of 51 sera (80\%) were not significantly affected by the GAD65-E517P perturbation, with results for binding to the mutant GAD65 falling above the $53 \%$ cut-off value. This frequency was significantly different from that for the Type 1 diabetes patients $(p<0.0001)$. This suggests that GAD65Ab in the low-risk general population are not directed against the conformational GAD65Ab epitope that is perturbed by the GAD65-E517P mutation. Similarly, sera from four SMS patients showed little reduction in binding to GAD65-E517P (mean binding $97 \pm 5 \%, 3 \%$ reduction) (Fig. 3a), regardless of serum dilution (Fig. 3b).

Sensitivity of GAD65Ab to GAD65-E517P versus $I A-2 A b$ positivity. Standard determination of risk of progression to Type 1 diabetes in at-risk subjects involves the measurement of multiple islet antibodies, including IA-2Ab, ICA, IAA and GAD65Ab, with multiple autoantibody positivity conferring a significantly increased risk compared with isolated single autoantibody positivity [28]. To better understand how a decrease in binding to GAD65-E517P compares with conventional measures of risk, we measured $\mathrm{IA}-2 \mathrm{Ab}$ in the first-degree relatives who progressed to Type 1 diabetes and in those who did not (Table 4). Among the non-progressors, binding to GAD65E517P was significantly reduced in the majority of individuals $(84 \%)$. Half of these individuals also tested positive for IA-2Ab (47\%). Conversely, among nonprogressors with no significant decrease in GAD65E517P binding, four demonstrated weak IA-2Ab positivity and two were IA-2Ab-negative. The vast majority of the progressors (92\%) demonstrated decreased binding to GAD65-E517P; however, only 64\% of these individuals were IA-2Ab positive. Thus, measuring reactivity with GAD65-E517P conferred increased sensitivity, but decreased specificity compared with measuring IA-2Ab, resulting in similar positive predictive values (GAD65-E517P: sensitivity 92\%, specificity $16 \%$, positive predictive value $26 \%$; IA-2Ab: sensitivity $58 \%$, specificity $40 \%$, positive predictive value $27 \%$ ).

Prediction of secondary structure. Although the threedimensional basis of the GAD65-E517P mutation that differentiates GAD65Ab binding in diabetic patients from that in healthy individuals is unclear, secondary
Table 4. Sensitivity of GAD65Ab binding to GAD65-E517P compared with IA-2Ab positivity as a measure of risk of progression to Type 1 diabetes

\begin{tabular}{l}
\hline Non-progressors $(n=38)$ \\
GAD65-E517P bindinga \\
$>53 \%(n=6)$ \\
\\
$<53 \%(n=32)$ \\
IA-2Ab status \\
4 weakly IA-2Ab-positive \\
15 IA-2Ab-negative \\
8 weakly IA-2Ab-positive \\
7 strongly IA-2Ab-positive \\
11 IA-2Ab-negative \\
6 N/A
\end{tabular}

Progressors $(n=12)$

GAD65-E517P binding ${ }^{\mathrm{a}} \quad$ IA-2Ab status

$>53 \%(n=1)$

$<53 \%(n=11)$

$1 \mathrm{IA}-2 \mathrm{Ab}$-negative

7 strongly IA-2Ab-positive

4 IA-2Ab-negative

a Values are percentage binding to GAD65-E517P compared with binding to wild-type GAD65 (100\%). N/A, not available

structural prediction at the E517 region of GAD65/67 indicates that the E517P mutation has a significant effect. Short peptide sequences (from position 505 to 544) from GAD65, GAD67 and GAD65-E517P were analysed using the National Cancer Institute computer modelling system. As illustrated in Figure 4, both GAD65 and GAD67 peptide sequences form alphahelical structures, but the helix positions are different. Three motifs (I, II and III) were discernible in GAD65. Motif I was absent in GAD67. Interestingly, motif I was present in GAD65-E517A, but not in GAD65-GVP or GAD65-E517P. The local shift to a GAD67-like structure by the GAD65-E517P mutation may explain the markedly reduced binding of Type 1 diabetes-associated GAD65Ab.

\section{Discussion}

Our data suggest that a single amino acid substitution at the $\mathrm{C}$ terminus of GAD65 dramatically reduces GAD65Ab binding in newly diagnosed Type 1 diabetes patients, in LADA patients and in first-degree relatives who subsequently progressed to disease. This mutation reduces GAD65 Ab binding to a lesser degree in "non-progressor" relatives, and minimally in GAD65-positive healthy adult subjects. The critical question is whether the predictive value of GAD65Ab for Type 1 diabetes is improved by comparing the binding of wild-type GAD65 and GAD65-E517P. All 85 Type 1 diabetes sera showed significantly reduced binding to the GAD65-E517P mutant (Fig. 3a). The high diagnostic sensitivity would therefore remain unaffected when comparing binding to GAD65 with binding to GAD65-E517P. However, GAD65Ab positivity alone has a relatively low predictive value for Type 1 diabetes [29, 30]. The binding of GAD65Ab 


\author{
GAD65: cfwyippslrtledneermsrlskvapvikarmmeygttm \\ aaaaaa aaaaaaaaaaaaaaaaaaaa \\ I II and III \\ GAD67 : cfwyipQslrGVPdSPQrREKlHkvapKikaLmmeSgttm \\ aaaaaaa aaaaaaaaa \\ II III \\ GAD65 : cfwyippslrtledneermsrlskvapvikarmmeygttm \\ aaaaa aaaaaaaaaaaaaaaaaa \\ I II and III \\ GAD65-P: CfwyippslrtlPdneermsrlskvapvikarmmeygttm \\ aaaaaaaaa aaaaaaaa \\ II III \\ GAD65-GVP : cfwyippslrGVPdneermsrlskvapvikarmmeygttm \\ aaaaaaaa aaaaaaaa \\ II III
}

Fig. 4. Secondary structure prediction of peptide sequences of amino-acid positions 505 to 544 in GAD65 and GAD67. The GAD65 mutants GAD65-P (E517P) and GAD65-GVP (T515G, L516V and E517P) are shown with the GAD67 amino acid residues in capital letters. Alpha helix sequences are indicated as "a" and motifs are indicated by the roman numerals I, II and III. The single amino acid substitution in the GAD65-E517P mutation is sufficient to abolish the "I" alpha helix motif specific to GAD65

was affected by the perturbation of the E517P mutation in only ten (19\%) of the 51 GAD65Ab-positive healthy subjects. The 51 GAD65Ab-positive sera were identified in a study of 2157 healthy subjects [7] and represent a diagnostic specificity of $97.7 \%$. A coanalysis with wild-type GAD65 and GAD65-E517P would have excluded the 44 sera in which the GAD65-E517P mutant did not induce a reduction in binding, and would therefore have increased the diagnostic specificity to $99.5 \%$. Thus, analysis of antibody binding to GAD65-E517P could be used as an adjunct to improve specificity in GAD65Ab-positive individuals whose risk is unclear, such as healthy GAD65Abpositive control subjects identified in population screenings.

Evaluation of antibody binding to the GAD65E517P mutant may also be a relevant means of risk assessment in other GAD65Ab-positive phenotypes. LADA patients represent a phenotypically variable group of GAD65Ab-positive individuals. GAD65Ab positivity (specifically, C-terminal epitope-specific GAD65Ab) in LADA patients [16] has been shown in follow-up studies to predict insulin dependency [31, 32]. The sera of all of the LADA patients evaluated in the current study demonstrated reduced binding to the mutant GAD65. Thus, further evaluation of this population with the GAD65-E517P mutant would not have provided additional information; however, it is possible that the reduction in GAD65-E517P binding would be subject to greater variation in a larger group of LADA patients. Further work is needed to evaluate whether binding to GAD65-E517P could potentially differentiate between LADA patients at higher and lower risk of progression to insulin dependency.

A differential analysis between wild-type GAD65 and GAD65-E517P may be particularly useful in GAD65Ab-positive first-degree relatives. It is known that islet cell autoantibodies in first-degree relatives predict Type 1 diabetes in subjects who test positive for multiple antibody markers (GAD65Ab, IA-2Ab or IAA) $[10,28,33]$. Our data suggest that a differential analysis between wild-type GAD65 and GAD65E517P may also be useful to better distinguish those first-degree relatives who are likely to progress to Type 1 diabetes from those who are not likely to progress to the disease. Of note, the predictive value of the percentage binding to GAD65-E517P for progression to Type 1 diabetes was comparable to that for GAD65/IA-2 double autoantibody positivity, with the GAD65-E517P evaluation conferring greater sensitivity but lower specificity. Among those who progressed to Type 1 diabetes, only $58 \%$ were IA-2Ab-positive, whereas $92 \%$ were sensitive to the E517P mutant. Thus, the E517P mutant identified four IA-2Ab-negative individuals who were apparently at lower risk of disease progression based on IA-2Ab status. Conversely, the degree of binding to GAD65-E517P was extremely variable among the non-progressors, ranging from no reduction compared with binding to wild- 
type GAD65, to near-complete eradication of binding to the mutant molecule. The majority of non-progressors $(84 \%)$ demonstrated a decrease in binding that was below the $53 \%$ cut-off level, conferring a specificity for Type 1 diabetes of only 16\% among firstdegree relatives. Similarly, IA-2Ab positivity was variable among this group, but conferred a higher specificity for disease (40\%). All individuals with strongly positive IA-2Ab titres also demonstrated a significant reduction in binding to the E517P molecule. Conversely, not all individuals with significant reductions in GAD65-E517P binding demonstrated IA- $2 \mathrm{Ab}$ positivity. Given that $50 \%$ of the "non-progressors" demonstrate multiple autoantibody positivity (IA-2Ab plus GAD65Ab), it is very likely that this group includes individuals who are at high risk of progression to diabetes, but the follow-up period of this study was not long enough to make this assessment. One of the limitations of this analysis is the relatively short length and variability of follow-up in the group of non-progressors and the consequent inability to determine long-term outcome. Based on the sensitivity of the GAD65-E517P mutant for disease positivity, we speculate that some of the non-progressors who demonstrated decreased binding to GAD65-E517P would progress to diabetes if followed for longer. Thus, it will be critical to study a larger group of firstdegree relatives over a longer period of time to confirm these findings and to determine whether the specificity and predictive value of differential binding between wild-type GAD65 and GAD65-E517P improves as more of the non-progressors develop Type 1 diabetes.

In other GAD65Ab-positive phenotypes such as SMS, approximately $25 \%$ of these rare patients also develop Type 1 diabetes $[34,35]$. In the four non-diabetic SMS patients in this study, there was no difference in binding between wild-type GAD65 and GAD65-E517P, regardless of the antibody dilution (Fig. 3b). This observation supports previous studies indicating that the GAD65Ab epitopes in SMS are distinct from those in Type 1 diabetes [34, 36].

In addition to further defining risk among GAD65Ab-positive individuals, these studies also contribute to our understanding of the GAD65 epitopes that are important for binding by disease-specific antibodies. The observation that the E517A mutation did not reduce GAD65Ab binding supports the concept that the proline residue present in GAD67 induces a structural change that markedly reduces binding, not only by GAD65Ab-positive sera but also by the MICA and GAD6 monoclonal antibodies. Our data further demonstrate that the E517P mutation defines an antibody epitope that is also dependent on amino acid residues in the middle part of GAD65 upstream of the enzymatic active site, such as amino acid residues 245-449 [37]. The previous finding that MICA1-3 bind a C-terminal epitope $[12,37,38]$ is confirmed by our analysis. Furthermore, MICA2, which recognises a linear epitope mapped to residues 506 to 531 [37, 38], showed an almost complete lack of binding to GAD65-E517P. Our results therefore demonstrate that a single C-terminal amino acid substitution not only eliminates MICA2 binding, but also significantly reduces the binding by MICA1/3 and MICA4/6, in this order. Similarly, the E517P mutation significantly reduces binding by the GAD6 monoclonal antibody. This monoclonal antibody has previously been shown to react with a GAD65 epitope encompassing amino acid residues 529 to 585 [39]; however, our observation that GAD6 does not bind GAD65E517P questions the precision of the previous mapping of the GAD6 conformational epitope. Finally, the reduction in binding of GAD65-E517P by the sera of Type 1 diabetes patients suggests that this conclusion is also valid for patient sera that are thought to have GAD65 autoantibodies directed against multiple epitopes as a result of putative epitope spreading.

Taken together, our data indicate that the GAD65 E517 residue is a critical determinant for the binding of Type 1 diabetes-associated conformation-dependent GAD65Ab. The reduced binding of Type 1 diabetes sera to the mutant molecule is specific to a glutamic acid to proline substitution at residue 517, since substituting with alanine did not affect the binding. Our data support previous speculations and preliminary molecular modelling [12] suggesting that residues in the middle and $\mathrm{C}$-terminal portion of GAD65 contribute to a Type 1 diabetes-associated conformational antibody epitope. A recent study using mutations induced at positions N247 and L574 in the GAD65 protein also demonstrated a loss of binding by GAD65 monoclonal antibodies and patient sera [40], supporting the hypothesis that the Type 1 diabetes conformational GAD65Ab epitope is dependent on amino acid residues at distant locations. In conclusion, GAD65-E517P may prove to be a useful reagent for improving the predictive value of GAD65Ab for Type 1 diabetes.

Acknowledgements. C. Hampe and I. Kockum were supported by research fellowships from the Juvenile Diabetes Foundation International. C. Greenbaum is suppoted in part by grants from the Paul G. Allen Foundation Clinical Scholars Program and the Buse Diabetes Clinical Research Chair. This work was supported by grants from the National Institutes of Health (DK26190, DK53004, DK42654), the Juvenile Diabetes Foundation International and Arbetsmarknadens Försäkringsaktiebolag (AFA Agreement-linked Insurance Company). Some subjects were seen at the General Clinical Research Center of the University of Washington (NOIRR00037). We thank D. F. Steiner and C. Wilson for critical review of the manuscript. We also thank P. Gohlke and E. A. Rutledge for their assistance in preparing samples for DNA sequencing. We acknowledge the contribution of J. Palmer in directing the Seattle Family Study. 


\section{References}

1. Falorni A, Kockum I, Sanjeevi CB, Lernmark Å (1995) Pathogenesis of insulin-dependent diabetes mellitus. Baillieres Clin Endocrinol Metab 9:25-46

2. Karlsen AE, Hagopian WA, Grubin CE et al. (1991) Cloning and primary structure of a human islet isoform of glutamic acid decarboxylase from chromosome 10. Proc Natl Acad Sci USA 88:8337-8341

3. Grubin CE, Daniels T, Toivola B et al. (1994) A novel radioligand binding assay to determine diagnostic accuracy of isoform-specific glutamic acid decarboxylase antibodies in childhood IDDM. Diabetologia 37:344-350

4. Hampe CS, Hammerle LP, Bekris L et al. (2000) Recognition of glutamic acid decarboxylase (GAD) by autoantibodies from different GAD antibody-positive phenotypes. J Clin Endocrinol Metab 85:4671-4679

5. Bingley PJ, Bonifacio E, Mueller PW (2003) Diabetes antibody standardization program: First assay proficiency evaluation. Diabetes 52:1128-1136

6. Rolandsson O, Hägg E, Nilsson M, Hallmans G, Lernmark $\AA$ (2001) Prediction of diabetes by screening with body mass index, oral glucose tolerance test (OGT) and islet cell autoantibodies in a regional population. J Internal Med 249:279-288

7. Rolandsson O, Hägg E, Hampe C et al. (1999) Levels of glutamate decarboxylase (GAD65) and tyrosine phosphatase-like protein (IA-2) autoantibodies in the general population are related to glucose intolerance and body mass index. Diabetologia 42:555-559

8. Hao W, Davis C, Hirsch IB et al. (1999) Plasmapheresis and immunosuppression in stiff-man syndrome with type 1 diabetes: a two year study. J Neurology 246:731-735

9. Greenbaum CJ, Sears KL, Kahn SE, Palmer JP (1999) Relationship of beta-cell function and autoantibodies to progression and nonprogression of subclinical type 1 diabetes-Follow-up of the Seattle Family Study. Diabetes 48:170-175

10. Verge CF, Gianani R, Kawasaki E et al. (1996) Prediction of type 1 diabetes in first-degree relatives using a combination of insulin, GAD, and ICA512bdc/IA-2 autoantibodies. Diabetes 45:926-933

11. Richter W, Eiermann TH, Endl J et al. (1993) Human monoclonal islet specific autoantibodies share features of islet cell and $64 \mathrm{kDa}$ antibodies. Diabetologia 36:785790

12. Schwartz HL, Chandonia JM, Kash SF et al. (1999) Highresolution autoreactive epitope mapping and structural modeling of the $65 \mathrm{kDa}$ form of human glutamic acid decarboxylase. J Mol Biol 287:983-999

13. Daw K, Powers AC (1995) Two distinct glutamic acid decarboxylase auto-antibody specificities in IDDM target different epitopes. Diabetes 44:216-220

14. Daw K, Ujihara N, Atkinson M, Powers AC (1996) Glutamic acid decarboxylase autoantibodies in stiff-man syndrome and insulin-dependent diabetes mellitus exhibit similarities and differences in epitope recognition. J Immunol 156:818-825

15. Sohnlein P, Muller M, Syren K et al. (2000) Epitope spreading and a varying but not disease-specific GAD65 antibody response in Type 1 diabetes. The Childhood Diabetes in Finland Study Group. Diabetologia 43:210-217

16. Falorni A, Gambelunghe G, Forini F et al. (2000) Autoantibody recognition of $\mathrm{COOH}$-terminal epitopes of GAD65 marks the risk for insulin requirement in adult-onset diabetes mellitus. J Clin Endocrinol Metab 85:309-316
17. Richter W, Endl J, Eiermann TH et al. (1992) Human monoclonal islet cell antibodies from a patient with insulin-dependent diabetes mellitus reveal glutamate decarboxylase as the target antigen. Proc Natl Acad Sci USA 89:8467-8471

18. Falorni A, Ackefors M, Carlberg C et al. (1996) Diagnostic sensitivity of immunodominant epitopes of glutamic acid decarboxylase (GAD65) autoantibodies epitopes in childhood IDDM. Diabetologia 39:1091-1098

19. Hampe CS, Lundgren P, Daniels TL et al. (2001) A novel monoclonal antibody specific for the N-terminal end of GAD65. J Neuroimmunology 113:63-71

20. Chang YC, Gottlieb DI (1988) Characterization of the proteins purified with monoclonal antibodies to glutamic acid decarboxylase. J Neuroscience 8:2123-2130

21. Richter W, Shi Y, Bækkeskov S (1993) Autoreactive epitopes defined by diabetes-associated human monoclonal antibodies are localized in the middle and C-terminal domains of the smaller form of glutamate decarboxylase. Proc Natl Acad Sci USA 90:2832-2836

22. Ludvigsson J, Heding L, Liedén G, Marner B, Lernmark A (1983) Plasmapheresis in the initial treatment of insulindependent diabetes mellitus in children. $\mathrm{Br}$ Med $\mathrm{J}$ 286:176-178

23. Li L, Jiang J, Hagopian WA et al. (1995) Differential detection of rat islet and brain glutamic acid decarboxylase (GAD) isoforms with sequence-specific peptide antibodies. J Histochem Cytochem 43:53-59

24. Schranz DB, Bekris L, Landin O-M et al. (1998) A simple and rapid microSepharose assay for GAD65 and ICA512 autoantibodies in diabetes. J Immunol Methods 213:8797

25. Torn C, Landin Olsson M, Ostman J et al. (2000) Glutamic acid decarboxylase antibodies (GADA) is the most important factor for prediction of insulin therapy within 3 years in young adult diabetic patients not classified as type 1 diabetes on clinical grounds. Diabetes Metab Res Rev $16: 442-447$

26. Hampe CS, Ortqvist E, Rolandsson O et al. (1999) Speciesspecific autoantibodies in type 1 diabetes. J Clin Endocrinol Metab 84:643-648

27. Mire-Sluis AR, Das RG, Lernmark $\AA$ (2000) The World Health Organization International Collaborative Study for Islet Cell Antibodies. Diabetologia 43:1282-1292

28. Notkins AL, Lernmark $\AA$ (2001) Autoimmune type 1 diabetes: resolved and unresolved issues. J Clin Invest 108:1247-1252

29. Bingley PJ, Bonifacio E, Williams AJK et al. (1997) Prediction of IDDM in the general population: Strategies based on combinations of autoantibody markers. Diabetes 46:1701-1710

30. Hagopian WA, Sanjeevi CB, Kockum I et al. (1995) Glutamate decarboxylase-, insulin- and islet cell-antibodies and HLA typing to detect diabetes in a general populationbased study of Swedish children. J Clin Invest 95:15051511

31. Hagopian WA, Karlsen AE, Gottsater A et al. (1993) Quantitative assay using recombinant human islet glutamic acid decarboxylase (GAD-64) showed $64 \mathrm{~K}$ autoantibody positivity at onset predicts diabetes type. J Clin Invest 91:368-374

32. Turner R, Stratton I, Horton V et al. (1997) UKPDS 25: Autoantibodies to islet-cell cytoplasm and glutamic acid decarboxylase for prediction of insulin requirement in type 2 diabetes. UK Prospective Diabetes Study Group. Lancet 350:1288-1293 
33. Bingley PJ, Christie MR, Bonifacio E et al. (1994) Combined analysis of autoantibodies improves prediction of IDDM in islet cell antibody-positive relatives. Diabetes 43:1304-1310

34. Kim J, Namchuck M, Bugawan T et al. (1994) Higher autoantibody levels and recognition of a linear NH2-terminal epitope in the autoantigen GAD65, distinguish stiffman syndrome from insulin-dependent diabetes mellitus. J Exp Med 180:595-606

35. Solimena M, Folli F, Aparisi R, Pozza G, De Camilli P (1990) Autoantibodies to GABA-ergic neurons and pancreatic beta cells in stiff-man syndrome. $\mathrm{N}$ Engl $\mathrm{J}$ Med 322:1555-1560

36. Dinkel K, Meinck H, Jury KM, Karges W, Richter W (1998) Inhibition of gamma-aminobutyric acid synthesis by glutamic acid decarboxylase autoantibodies in stiff-man syndrome. Ann Neurol 44:194-201

37. Syren K, Lindsay L, Stoehrer B et al. (1996) Immune reactivity of diabetes-associated human monoclonal autoantibodies defines multiple epitopes and detects two domain boundaries in glutamate decarboxylase. J Immunol 157:5208-5214
38. Richter W, Northemann W, Müller M, Böhm BO (1996) Mapping of an autoreactive epitope with glutamate decarboxylase using a diabetes-associated monoclonal antibody and an epitope cDNA library. Hybridoma 15:103108

39. Powers AC, Eisenbarth GS (1985) Autoimmunity to islet cells in diabetes mellitus. Ann Rev Med 36:533-544

40. Tree TIM, Morgenthaler NG, Duhindan N et al. (2000) Two amino acids in glutamic acid decarboxylase act in concert for maintenance of conformational determinants recognised by type 1 diabetic autoantibodies. Diabetologia 43:881-889

41. Bonifacio E, Lernmark Å, Dawkins RL (1988) Serum exchange and use of dilutions have improved precision of measurement of islet cell antibodies. J Immunol Methods 106:83-88

42. Mire-Sluis AR, Das RG, Lernmark A (1999) The development of a World Health Organisation international standard for islet cell antibodies: The aims and design of an international collaborative study. Diabetes Metab Res Rev 15:7277 